\title{
Coronary Lesion Complexity
}

National Cancer Institute

\section{Source}

National Cancer Institute. Coronary Lesion Complexity. NCI Thesaurus. Code C100066.

Characteristics of coronary lesions that contribute to procedural risk during coronary intervention. 